\title{
Erratum: Head-tail modes for strong space charge [Phys. Rev. ST Accel. Beams 12, 044202 (2009)]
}

\author{
A. Burov \\ (Received 21 August 2009; published 12 October 2009) \\ PACS numbers: 29.20.db, 99.10.Cd
}

DOI: 10.1103/PhysRevSTAB.12.109901

A recently published paper [1] shows some disagreement with the author's article [2], hereafter referred to as "the article." Checking my derivation of Eq. (25) in the article, I found an error in Eq. (24). The second derivative $d^{2} \bar{y} / d \Psi^{2}$ on the right-hand side of Eq. (24) should be calculated as follows:

$$
\begin{aligned}
\frac{d^{2} \bar{y}}{d \Psi^{2}} & =\frac{d}{d \Psi}\left(\frac{\dot{\tau}}{Q(\tau)} \frac{\partial}{\partial \tau}-i \frac{\nu}{Q(\tau)}\right) \bar{y} \approx \frac{d}{d \Psi}\left(\frac{\dot{\tau}}{Q(\tau)} \frac{d \bar{y}}{d \tau}\right)=\frac{\dot{\tau}^{2}}{Q(\tau)} \frac{d}{d \tau}\left(\frac{1}{Q(\tau)} \frac{d \bar{y}}{d \tau}\right)+\frac{\ddot{\tau}}{Q^{2}(\tau)} \frac{d \bar{y}}{d \tau} \\
& \equiv \frac{v^{2}}{Q(\tau)} \frac{d}{d \tau}\left(\frac{1}{Q(\tau)} \frac{d \bar{y}}{d \tau}\right)+\frac{F(\tau)}{Q^{2}(\tau)} \frac{d \bar{y}}{d \tau} .
\end{aligned}
$$

Here $\ddot{\tau}=F(\tau)$ is the local longitudinal acceleration (force) in the given rf potential well. The article mistakenly assumes the commutation of the single-particle time derivative and ensemble averaging. These quantities do not generally commute. Subsequent averaging yields

$$
\nu \bar{y}+\frac{F(\tau)}{Q(\tau)} \frac{d \bar{y}}{d \tau}+u^{2}(\tau) \frac{d}{d \tau}\left(\frac{1}{Q(\tau)} \frac{d \bar{y}}{d \tau}\right)=0 .
$$

For a linear force assumed in Ref [1], $F(\tau)=-Q_{s}^{2} \tau$, this result agrees with Eq. (26) of Ref [1]. For arbitrary rf bucket and steady state distribution

$$
F-\frac{u^{2}}{Q} \frac{d Q}{d \tau}=\frac{d u^{2}}{d \tau}
$$

leading to

$$
\frac{d}{d \tau}\left(u^{2} \frac{d \bar{y}}{d \tau}\right)+\nu Q \bar{y}=0 .
$$

Equation (25) of the article must be disregarded, and Eq. (3) above should be used instead. This equation is also self-adjoint and with the zero-derivative boundary condition $\bar{y}^{\prime}( \pm \infty)=0$ [Eq. (30) of the article] yields orthogonality of the eigenfunctions:

$$
\int_{-\infty}^{\infty} d \tau \rho(\tau) \bar{y}_{i}(\tau) \bar{y}_{j}(\tau)=\delta_{i j} N_{b}
$$

This orthogonality relation differs from the corresponding result of the article by a substitution

$$
u^{-1}(\tau) \rightarrow \rho(\tau) / N_{b}=Q(\tau) / \int d \tau Q(\tau)
$$

thus, this substitution must be used for corrections of the coherent tune shifts of Eq. (32), and the wake matrix elements of Eq. (60) in the article. Integral rules $(15,27)$ of Ref. [1] for the selection of eigenvalues follow from Eq. (3) together with the zero-derivative boundary condition.

For a square potential well, Eq. (3) above is identical to Eq. (25) of the article, so for this case no correction is needed. For a Gaussian bunch, Eq. (3) yields

$$
\bar{y}^{\prime \prime}+\nu \exp \left(-\tau^{2} / 2\right) \bar{y}=0,
$$

which must be used instead of Eq. (36) of the article, with the same units. The first five solutions are presented in Fig. 1 below, correcting Fig. 2 of the article.

The asymptotic behavior of the eigenfunction derivative is important for the Landau damping rate; according to Eq. (5),

$$
\bar{y}^{\prime}(\tau) \cong \nu \exp \left(-\tau^{2} / 2\right) y_{\infty} / \tau ; \quad y_{\infty} \equiv \bar{y}(\infty) .
$$




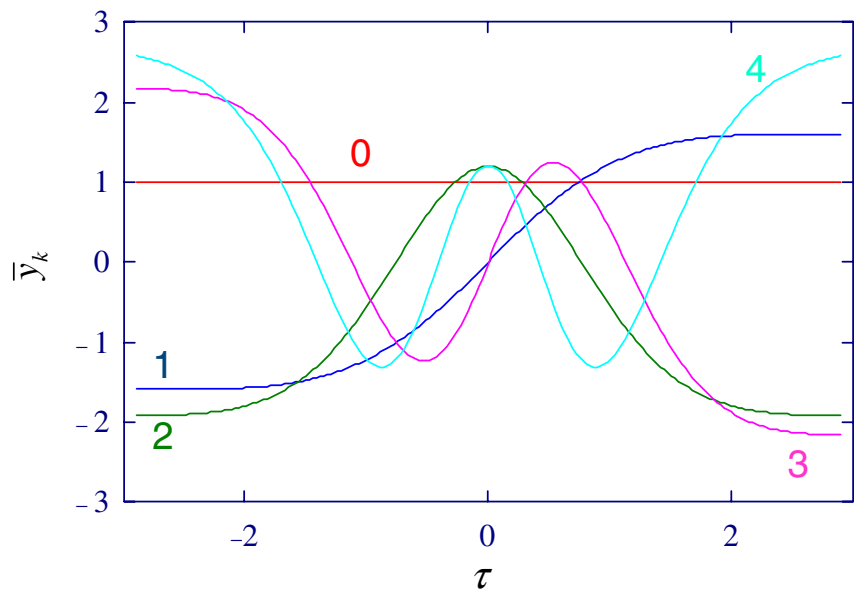

FIG. 1. (Color) Eigenfunctions of the Gaussian bunch.

The numerically determined first ten eigenvalues $\nu_{k}$, with eigenfunction asymptotes $\left|y_{\infty}\right|$, are presented in Table I below, which corrects Table I of the article.

Substituting this corrected asymptotic formula in the general result for intrinsic Landau damping, Eq. (45b) of the article, yields

$$
\Lambda_{k}=0.5 \frac{\nu_{k}^{2} y_{\infty k}^{2}}{\tau_{*}} \frac{Q_{s}}{q^{3}} \sim k^{4} \frac{Q_{s}}{q^{3}} .
$$

The factor $A$ in Eq. (56) of the article should be corrected to $A \approx 25$, while the asymptotic values $\left|y_{\infty}\right|$ should be taken from Table I above.

Equations (57) of the article are corrected according to

$$
\begin{gathered}
\nu \bar{y}(\tau)+\frac{1}{Q_{\mathrm{eff}}(\tau)} \frac{d}{d \tau}\left(u^{2} \frac{d \bar{y}}{d \tau}\right)=\kappa(\hat{\mathbf{W}} \bar{y}+\hat{\mathbf{D}} \bar{y}) \quad \hat{\mathbf{W}} \bar{y} \equiv \int_{\tau}^{\infty} W(\tau-s) \exp [i \zeta(\tau-s)] \rho(s) \bar{y}(s) d s ; \\
\hat{\mathbf{D}} \bar{y} \equiv \bar{y}(\tau) \int_{\tau}^{\infty} D(\tau-s) \rho(s) d s .
\end{gathered}
$$

TABLE I. Eigenvalues and asymptotic values for the first ten modes of the Gaussian bunch.

\begin{tabular}{lcccccccccc}
\hline \hline$k$ & 0 & 1 & 2 & 3 & 4 & 5 & 6 & 7 & 8 & 9 \\
\hline$\nu$ & 0 & 1.4 & 4.4 & 8.9 & 15 & 23 & 32 & 43 & 56 & 70 \\
$\left|y_{\infty}\right|$ & 1 & 1.6 & 2.0 & 2.3 & 2.5 & 2.7 & 2.9 & 3.0 & 3.2 & 3.3 \\
\hline \hline
\end{tabular}

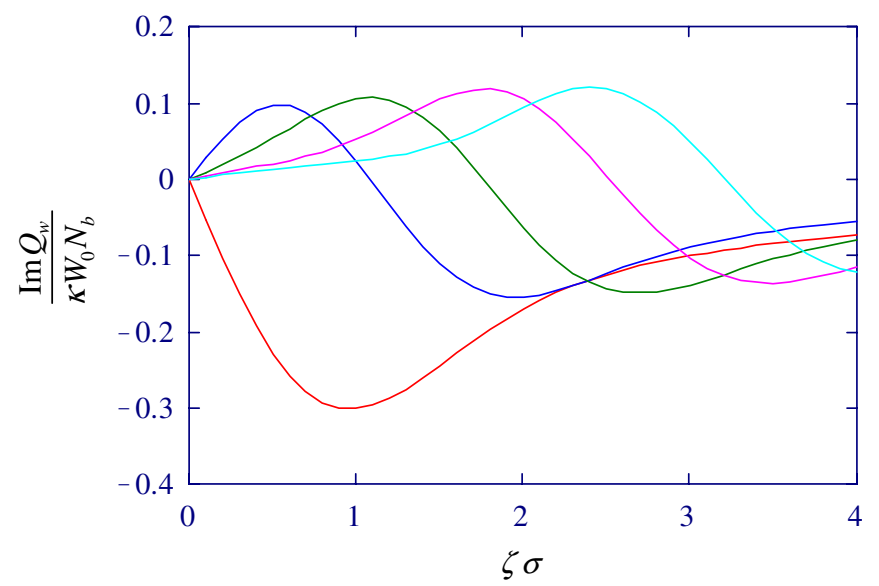

FIG. 2. (Color) Coherent growth rates for the Gaussian bunch with the constant wake. 


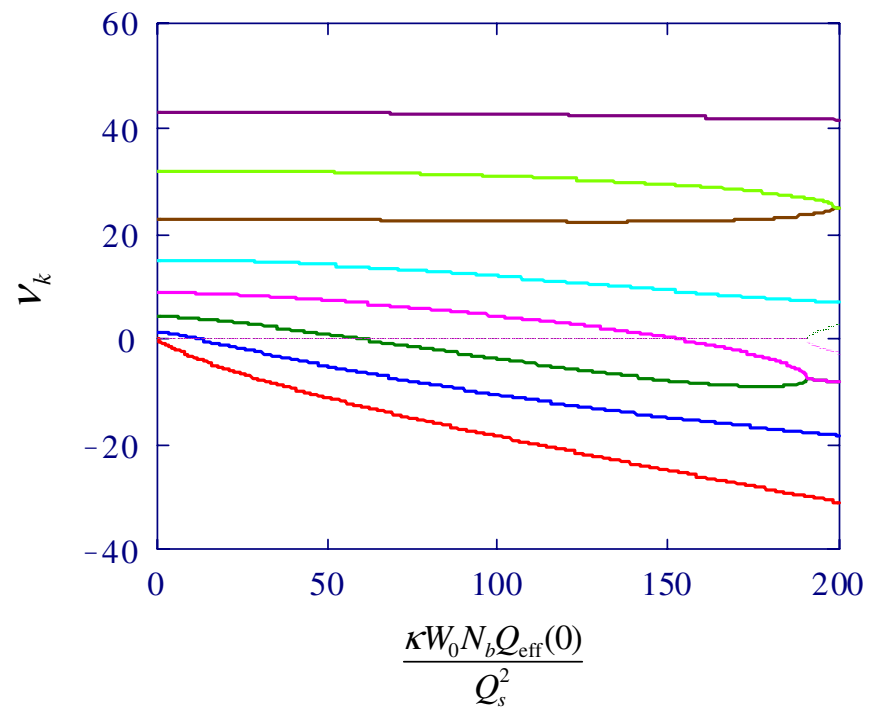

FIG. 3. (Color) Coherent tunes of the Gaussian bunch for constant wake, no detuning, and zero chromaticity.

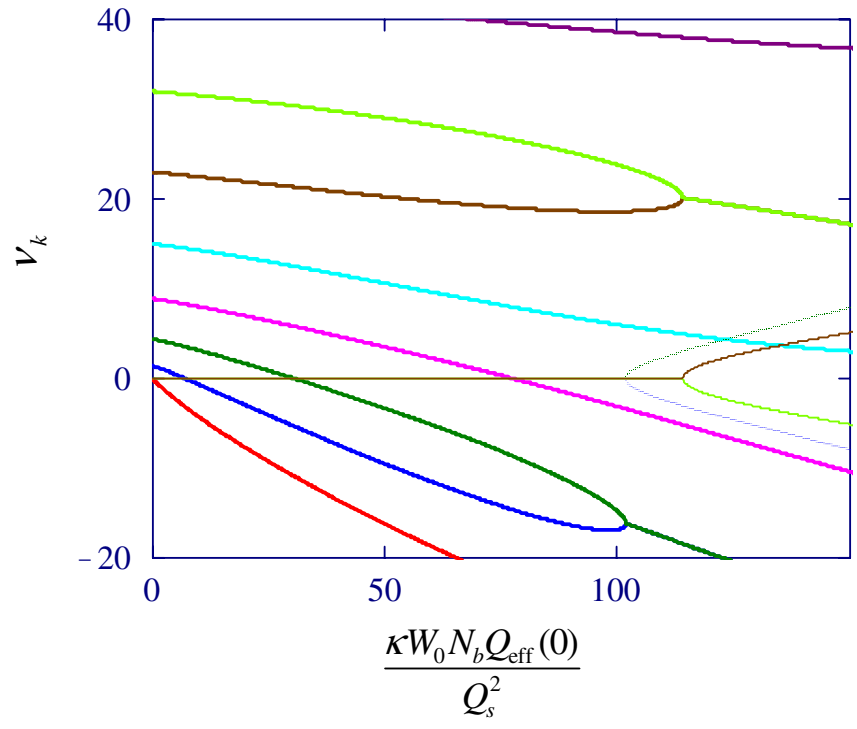

FIG. 4. (Color) Same as Fig. 3, but for the resistive wake.

This yields the matrix element correction of Eq. (60):

$$
\begin{aligned}
\hat{\mathbf{W}}_{k m} & =N_{b}^{-1} \int_{-\infty}^{\infty} \int_{\tau}^{\infty} W(\tau-s) \exp [i \zeta(\tau-s)] \rho(s) \rho(\tau) \bar{y}_{0 k}(\tau) \bar{y}_{0 m}(s) d s d \tau, \\
\hat{\mathbf{D}}_{k m} & =N_{b}^{-1} \int_{-\infty}^{\infty} \int_{\tau}^{\infty} D(\tau-s) \rho(s) \rho(\tau) \bar{y}_{0 k}(\tau) \bar{y}_{0 m}(\tau) d s d \tau .
\end{aligned}
$$

Figures 2-4 below correct Figs. 3, 4, and 5, respectively, of the article. The changes do not appear to be significant.

[1] V. Balbekov, Report No. FERMILAB-PUB-09-322, 2009.

[2] A. Burov, Phys. Rev. ST Accel. Beams 12, 044202 (2009). 